TAIWANESE JOURNAL OF MATHEMATICS

Vol. 19, No. 1, pp. 175-191, February 2015

DOI: $10.11650 /$ tjm.19.2015.4447

This paper is available online at http://journal.taiwanmathsoc.org.tw

\title{
PARACONTACT METRIC MANIFOLDS WITHOUT A CONTACT METRIC COUNTERPART
}

\author{
Verónica Martín-Molina
}

\begin{abstract}
We study non-paraSasakian paracontact metric $(\kappa, \mu)$-spaces with $\kappa=$ -1 (equivalent to $h^{2}=0$ but $h \neq 0$ ). These manifolds, which do not have a contact geometry counterpart, will be classified locally in terms of the rank of $h$. We will also give explicit examples of every possible constant rank of $h$.
\end{abstract}

\section{INTRODUCTION}

A remarkable class of paracontact metric manifolds $(M, \phi, \xi, \eta, g)$ is that of paracontact metric $(\kappa, \mu)$-spaces, which satisfy the nullity condition

$$
R(X, Y) \xi=\kappa(\eta(Y) X-\eta(X) Y)+\mu(\eta(Y) h X-\eta(X) h Y),
$$

for all $X, Y$ vector fields on $M$, where $\kappa$ and $\mu$ are constants and $h=\frac{1}{2} L_{\xi} \varphi$.

This definition, which may appear quite technical, arises from the deep and meaningful relationship between contact metric $(\kappa, \mu)$-spaces and paracontact geometry. More precisely, it was proved in [7] that any non-Sasakian contact metric $(\kappa, \mu)$ space accepts two paracontact metric $(\widetilde{\kappa}, \widetilde{\mu})$-structures with the same contact form. On the other hand, under certain natural conditions, every non-paraSasakian paracontact $(\widetilde{\kappa}, \widetilde{\mu})$-space carries a contact metric $(\kappa, \mu)$-structure compatible with the same contact form ([8]). The class of paracontact metric $(\kappa, \mu)$-spaces includes the paraSasakian ones (see [12] and [15]) and the ones satisfying $R(X, Y) \xi=0$ for all $X, Y$ (studied in [16]), among others.

There are some notable differences between a contact metric $(\kappa, \mu)$-space $(M, \phi, \xi$, $\eta, g)$ and a paracontact metric $(\widetilde{\kappa}, \widetilde{\mu})$-space $(\widetilde{M}, \widetilde{\phi}, \widetilde{\xi}, \widetilde{\eta}, \widetilde{g})$. First of all, while they

Received February 18, 2014, accepted May 15, 2014.

Communicated by Bang-Yen Chen.

2010 Mathematics Subject Classification: 53C15, 53C25, 53C50.

Key words and phrases: Paracontact metric manifold, ParaSasakian, Nullity distribution, $(\kappa, \mu)$-spaces.

The author is is partially supported by the PAI group FQM-327 (Junta de Andalucía, Spain), the group Geometría E15 (Gobierno de Aragón, Spain), the MINECO grant MTM2011-22621 and the "Centro Universitario de la Defensa de Zaragoza" grant ID2013-15. 
satisfy $h^{2}=(\kappa-1) \varphi^{2}$ and $\widetilde{h}^{2}=(\widetilde{\kappa}+1) \widetilde{\varphi}^{2}$, respectively, the first condition means that $\kappa \leq 1$ but the second one does not give any type of restriction for $\widetilde{\kappa}$ because the metric of a paracontact metric manifold is not positive definite (see [2] for the contact metric case and [8] for the paracontact metric one).

Another difference is that, in the contact metric case, $\kappa=1$ (i.e. $h^{2}=0$ ) is also equivalent to the manifold being Sasakian (and thus $h=0$ ). However, there are paracontact metric $(\widetilde{\kappa}, \widetilde{\mu})$-spaces with $\widetilde{\kappa}=-1$ (and thus $\widetilde{h}^{2}=0$ ) but $\widetilde{h} \neq 0$. The first example of paracontact metric $(-1,2)$-space $\left(M^{2 n+1}, \widetilde{\phi}, \widetilde{\xi}, \widetilde{\eta}, \widetilde{g}\right)$ with $\widetilde{h} \neq 0$ was given in [5] for $n=2$. Later, an example with arbitrary $n$ appeared in [8] (constructed by deforming the contact metric structure defined on the unit tangent sphere bundle) and a numerical example with $n=1$ was shown in [13]. It is worth mentioning that all these spaces have $\widetilde{\mu}=2$ and $\operatorname{rank}(\widetilde{h})=n$. Lastly, an example of 3-dimensional paracontact metric $(-1,0)$-space with $\widetilde{h} \neq 0$ appeared in [10].

To our knowledge, no effort has been made to better understand the general behaviour of the tensor $h$ of a paracontact metric $(\kappa, \mu)$-space when $h^{2}=0$ but $h \neq 0$, which we will address in Theorem 3.2. We will study the form of the tensor $h$ in this remarkable situation and we will later construct explicit examples that illustrate all the possible constant values of the rank of $h$ (from 1 to $n$ ) when $\mu=2$. Finally, we will discuss the situation when $\mu \neq 2$ and show some paracontact metric $(-1,0)$-spaces with $h \neq 0$ in Examples 3.8-3.11. These are the first examples of this type with $\mu \neq 2$ and dimension greater than 3 .

\section{Preliminaries}

An almost paracontact structure on a $(2 n+1)$-dimensional smooth manifold $M$ is given by a $(1,1)$-tensor field $\varphi$, a vector field $\xi$ and a 1-form $\eta$ satisfying the following conditions [12]:

(i) $\eta(\xi)=1, \varphi^{2}=I-\eta \otimes \xi$,

(ii) the eigendistributions $\mathcal{D}^{+}$and $\mathcal{D}^{-}$of $\varphi$ corresponding to the eigenvalues 1 and -1 , respectively, have equal dimension $n$.

As an immediate consequence, $\varphi \xi=0, \eta \circ \varphi=0$ and the tensor $\varphi$ has constant rank $2 n$. If an almost paracontact manifold is endowed with a semi-Riemannian metric $g$ such that

$$
g(\varphi X, \varphi Y)=-g(X, Y)+\eta(X) \eta(Y),
$$

for all $X, Y$ on $M$, then $(M, \varphi, \xi, \eta, g)$ is called an almost paracontact metric manifold. Note that such a semi-Riemannian metric is necessarily of signature $(n+1, n)$ and the above condition (ii) of the definition of almost paracontact structures is automatically satisfied. Moreover, it follows easily that $\eta=g(\cdot, \xi)$ and $g(\cdot, \varphi \cdot)=-g(\varphi \cdot, \cdot)$. We can now define the fundamental 2 -form of the almost paracontact metric manifold by 
$\Phi(X, Y)=g(X, \varphi Y)$. If $d \eta=\Phi$, then $\eta$ becomes a contact form (i.e. $\eta \wedge(d \eta)^{n} \neq 0$ ) and $(M, \varphi, \xi, \eta, g)$ is said to be a paracontact metric manifold.

We can also define on a paracontact metric manifold the tensor field $h:=\frac{1}{2} L_{\xi} \varphi$, which is a symmetric operator with respect to $g$, anti-commutes with $\varphi$ and satisfies $h \xi=\operatorname{tr} h=0$ and the identity $\nabla \xi=-\varphi+\varphi h$ ([15]). Moreover, it vanishes identically if and only if $\xi$ is a Killing vector field, in which case $(M, \varphi, \xi, \eta, g)$ is called a K-paracontact manifold.

An almost paracontact structure is said to be normal if and only if the tensor $N_{\varphi}-2 d \eta \otimes \xi$ vanishes identically, where $N_{\varphi}$ is the Nijenhuis tensor of $\varphi: N_{\varphi}(X, Y)=$ $[\varphi, \varphi](X, Y)=\varphi^{2}[X, Y]+[\varphi X, \varphi Y]-\varphi[\varphi X, Y]-\varphi[X, \varphi Y]$ ([15]). A normal paracontact metric manifold is said to be a paraSasakian manifold and satisfies

$$
R(X, Y) \xi=-(\eta(Y) X-\eta(X) Y),
$$

for every $X, Y$ on $M$. Unlike in the contact metric case, the condition (2.1) does not imply that the manifold is paraSasakian, as will be seen in Examples 3.8-3.11.

It was also proved in [15] that an almost paracontact manifold is paraSasakian if and only if

$$
\left(\nabla_{X} \varphi\right) Y=-g(X, Y) \xi+\eta(Y) X
$$

so, in particular, every paraSasakian manifold is $K$-paracontact. The converse holds in dimension 3 ([9]) and for $(-1, \mu)$-spaces (which will be proved in Theorem 3.1) but we can construct explicit examples that show that these two concepts are not equivalent in general.

Example 2.1. Let $\mathfrak{g}$ be the 5-dimensional Lie algebra with basis $\left\{\xi, X_{1}, Y_{1}, X_{2}, Y_{2}\right\}$ such that the only non-vanishing Lie brackets are

$$
\left[X_{1}, Y_{1}\right]=2 \xi, \quad\left[X_{2}, Y_{2}\right]=2 \xi,\left[X_{1}, X_{2}\right]=Y_{1} .
$$

If we denote by $G$ the Lie group whose Lie algebra is $\mathfrak{g}$, we can define a left-invariant paracontact metric structure on $G$ the following way:

$$
\begin{gathered}
\varphi \xi=0, \quad \varphi X_{i}=X_{i}, \varphi Y_{i}=-Y_{i}, \eta(\xi)=1, \quad \eta\left(X_{i}\right)=\eta\left(Y_{i}\right)=0, \quad i=1,2, \\
g(\xi, \xi)=g\left(X_{1}, Y_{1}\right)=g\left(X_{2}, Y_{2}\right)=1, \\
g\left(\xi, X_{i}\right)=g\left(\xi, Y_{i}\right)=g\left(X_{i}, X_{j}\right)=g\left(Y_{i}, Y_{j}\right)=0, \quad i=1,2 .
\end{gathered}
$$

A simple computation gives that $h=0$, so the manifold is $K$-paracontact.

However, although $R(X, \xi) \xi=-X$ for all vector field $X$ orthogonal to $\xi$, straightforward computations give that $R\left(X_{1}, X_{2}\right) \xi=-2 Y_{1} \neq 0$, so the manifold does not satisfy (2.1). In particular, it is not paraSasakian. 
Finally, we recall that a notion of $\mathcal{D}_{c}$-homothetic deformation can be introduced in paracontact metric geometry ([15]). Indeed, given a non-zero constant $c$, a $\mathcal{D}_{c^{-}}$ homothetic deformation on a paracontact metric manifold $(M, \varphi, \xi, \eta, g)$ is the following change of the structure tensors:

$$
\varphi^{\prime}:=\varphi, \quad \xi^{\prime}:=\frac{1}{c} \xi, \quad \eta^{\prime}:=c \eta, \quad g^{\prime}:=c g+c(c-1) \eta \otimes \eta .
$$

Then $\left(\varphi^{\prime}, \xi^{\prime}, \eta^{\prime}, g^{\prime}\right)$ is again a paracontact metric structure on $M$. Moreover, $K$ paracontact and paraSasakian structures are also preserved under $\mathcal{D}_{c}$-homothetic deformations.

Although $D_{c}$-homothetic deformations destroy curvature conditions like $R(X, Y) \xi=$ 0 , a $D_{c}$-homothetically deformed paracontact metric $(\kappa, \mu)$-space is another paracontact metric $\left(\kappa^{\prime}, \mu^{\prime}\right)$-space with

$$
\kappa^{\prime}=\frac{\kappa+1-c^{2}}{c^{2}}, \quad \mu^{\prime}=\frac{\mu-2+2 c}{c} .
$$

\section{Paracontact Metric $(-1, \mu)$-Spaces}

We now present our main results.

Theorem 3.1. Let $M$ be a paracontact metric manifold such that $R(X, Y) \xi=$ $-(\eta(Y) X-\eta(X) Y)$, for all $X, Y$ on $M$. Then $M$ is paraSasakian if and only if $\xi$ is a Killing vector field.

Proof. If $M$ is paraSasakian, it is in particular a $K$-paracontact manifold, hence $\xi$ is a Killing vector field.

Conversely, if $\xi$ is a Killing vector field, then $h=0$ and $\nabla_{X} \xi=-\varphi X$ holds for every $X$ on $M$ ([15]). We also know from [14, p.259] that

$$
R(\xi, X) Y=-\nabla_{X} \nabla_{Y} \xi+\nabla_{\nabla_{X} Y} \xi
$$

thus $R(\xi, X) Y=\left(\nabla_{X} \varphi\right) Y$, for all $X, Y$ on $M$.

Therefore, it follows from the previous formula and from $R(X, Y) \xi=-(\eta(Y) X-$ $\eta(X) Y)$ that

$g\left(\left(\nabla_{X} \varphi\right) Y, Z\right)=g(R(\xi, X) Y, Z)=g(R(Y, Z) \xi, X)=g(-g(X, Y) \xi+\eta(Y) X, Z)$, hence equation (2.2) holds and the manifold is paraSasakian.

Theorem 3.2. Let $M$ be a $(2 n+1)$-dimensional paracontact metric $(-1, \mu)$-space. Then we have one of the following possibilities:

(1) $h=0$ and $M$ is paraSasakian 
(2) $h \neq 0$ and $\operatorname{rank}\left(h_{p}\right) \in\{1, \ldots, n\}$ at every $p \in M$ where $h_{p} \neq 0$. Moreover, there exists a basis $\left\{\xi, X_{1}, Y_{1}, \ldots, X_{n}, Y_{n}\right\}$ of $T_{p}(M)$ such that

- The only non-vanishing components of $g$ are

$$
g_{p}(\xi, \xi)=1, \quad g_{p}\left(X_{i}, Y_{i}\right)= \pm 1
$$

- and

$$
h_{\mid\left\langle X_{i}, Y_{i}\right\rangle}=\left(\begin{array}{cc}
0 & 0 \\
1 & 0
\end{array}\right) \quad \text { or } \quad h_{\mid\left\langle X_{i}, Y_{i}\right\rangle}=\left(\begin{array}{ll}
0 & 0 \\
0 & 0
\end{array}\right)
$$

where obviously there are exactly rank $\left(h_{p}\right)$ submatrices of the first type.

If $n=1$, such a basis $\left\{\xi, X_{1}, Y_{1}\right\}$ also satisfies that

$$
\varphi X_{1}= \pm X_{1}, \quad \varphi Y_{1}=\mp Y_{1}
$$

and the tensor $h$ can be written as

$$
h_{\mid\left\langle\xi, X_{i}, Y_{i}\right\rangle}=\left(\begin{array}{ccc}
0 & 0 & 0 \\
0 & 0 & 0 \\
0 & 1 & 0
\end{array}\right) .
$$

Proof. We know from Lemma 3.2 of [8] that $h^{2}=0$. We have now two possibilities.

If $h=0$, then $R(X, Y) \xi=-(\eta(Y) X-\eta(X) Y)$, for all $X, Y$ on $M$ and $\xi$ is a Killing vector field. Therefore, it follows from Theorem 3.1 that the manifold is paraSasakian.

Let us now suppose that $h \neq 0$. Since $h$ is self-adjoint and $\operatorname{Ker}(\eta)$ is $h$-invariant, we have from [14, p.260] that, at each point $p \in M, \operatorname{Ker}\left(\eta_{p}\right)=V_{1} \oplus \cdots \oplus V_{l}$ (for some $1 \leq l \leq 2 n$ ), where $V_{k}$ are mutually orthogonal subspaces that are $h$-invariant and on which $h_{\mid V_{k}}$ has a matrix of either type:

$$
\left(\begin{array}{ccccc}
\lambda & & & & \\
1 & \lambda & & \mathbf{0} & \\
& 1 & \lambda & & \\
& & \ddots & \ddots & \\
& 0 & & 1 & \lambda
\end{array}\right)
$$

relative to a basis $X_{1}, \ldots, X_{r}$ of $V_{k}, r \geq 1$, such that the only non-zero products are 
$g_{p}\left(X_{i}, X_{j}\right)= \pm 1$ if $i+j=r+1$, or of type

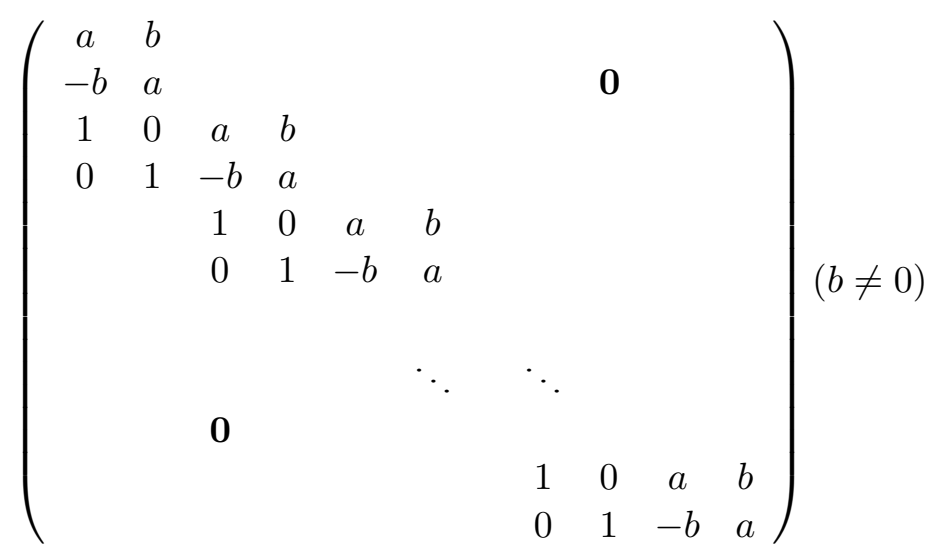

relative to a basis $X_{1}, Y_{1}, \ldots, X_{m}, Y_{m}$ of $V_{k}$, such that the only non-zero products are $g_{p}\left(X_{i}, X_{j}\right)=1=-g_{p}\left(Y_{i}, Y_{j}\right)$ if $i+j=m+1$.

We will first see that the second case is not possible. Indeed, if there existed a subspace $V_{k}$ such that $h_{\mid V_{k}}$ had a matrix of type (3.3), then $h_{p}^{2} X_{1}=\left(a^{2}-b^{2}\right) X_{1}-$ $2 a b Y_{1}+2 a X_{2}-2 b Y_{2}+X_{3}=0$, which cannot happen for any value of $m$ because $b \neq 0$.

If there exists a subspace $V_{k}$ such that $h_{\mid V_{k}}$ has a matrix of type (3.2), then $h_{p}^{2} X_{1}=$ $\lambda^{2} X_{1}+2 \lambda X_{2}+X_{3}=0$, which is only possible if $\lambda=0$ and $\operatorname{dim} V_{k}=r \leq 2$. Let us distinguish between both subcases:

(1) If $\operatorname{dim} V_{i}=2$, then $V_{i}=\left\langle X_{i}, Y_{i}\right\rangle$, the only non-zero product is $g_{p}\left(X_{i}, Y_{i}\right)= \pm 1$ and

$$
h_{\mid\left\langle X_{i}, Y_{i}\right\rangle}=\left(\begin{array}{cc}
0 & 0 \\
1 & 0
\end{array}\right)
$$

(2) If $\operatorname{dim} V_{i}=1$, then $V_{i}=\left\langle X_{i}\right\rangle$ and $h_{p} X_{i}=0$, with $X_{i}$ a vector satisfying $g_{p}\left(X_{i}, X_{i}\right)= \pm 1$. In fact, since $\operatorname{Ker}\left(\eta_{p}\right)$ is of signature $(n, n)$ and the subspaces $V_{i}$ of the subcase (1) are of dimension two and signature $(1,1)$, then there is an even number of subspaces of this type, half satisfying $g_{p}\left(X_{i}, X_{i}\right)=1$ and half satisfying $g_{p}\left(X_{i}, X_{i}\right)=-1$. Taking one of each type, for example $V_{1}=\left\langle X_{1}\right\rangle$ and $V_{2}=\left\langle X_{2}\right\rangle$ with $g_{p}\left(X_{1}, X_{1}\right)=1=-g_{p}\left(X_{2}, X_{2}\right)$, a simple change of basis like $\widetilde{X_{1}}=\frac{1}{\sqrt{2}}\left(X_{1}+X_{2}\right)$ and $\widetilde{Y_{1}}=\frac{1}{\sqrt{2}}\left(X_{1}-X_{2}\right)$ would give us a basis such that the only non-vanishing component of the metric is $g_{p}\left(\widetilde{X}_{1}, \widetilde{Y}_{1}\right)=1$.

Finally, since $\left.h\right|_{V_{i}}=0$ in the second subcase, the rank of $h_{p}$ depends on the number of submatrixes of the first type, which have rank 1 . Therefore, $\operatorname{rank}\left(h_{p}\right) \in\{1, \ldots, n\}$ at every point where $h_{p} \neq 0$. 
In dimension 3 , the conditions over $\varphi$ are the only ones that remain to be proved. First of all, $\varphi \xi=0$ on every paracontact metric structure. Hence $\varphi X_{1}=a X_{1}+b Y_{1}$, for some constants $a, b$.

It follows from $g_{p}\left(X_{1}, \varphi X_{1}\right)=d \eta_{p}\left(X_{1}, X_{1}\right)=0$ that $b=0$, so $\varphi X_{1}=a X_{1}$. On the other hand, $\varphi Y_{1}=\varphi h X_{1}=-h \varphi X_{1}=-a h X_{1}=-a Y_{1}$, hence $\varphi Y_{1}=-a Y_{1}$. We can then compute:

$$
\begin{aligned}
& g_{p}\left(\varphi X_{1}, \varphi Y_{1}\right)=-g_{p}\left(X_{1}, Y_{1}\right)=\mp 1, \\
& g_{p}\left(\varphi X_{1}, \varphi Y_{1}\right)=g_{p}\left(a X_{1},-a Y_{1}\right)=-a^{2} g_{p}\left(X_{1}, Y_{1}\right)=\mp a^{2},
\end{aligned}
$$

so $a^{2}=1$, and thus

$$
\varphi X_{1}= \pm X_{1}, \quad \varphi Y_{1}=\mp Y_{1},
$$

which ends the proof.

Remark 3.3. A paracontact metric manifold satisfying

$$
R(X, Y) \xi=-(\eta(Y) X-\eta(X) Y)
$$

for all $X, Y$ on $M$, can be either a $(-1, \mu)$-space with $h=0$ (thus paraSasakian by the previous Theorem and $\mu$ is undetermined) or a paracontact metric $(-1,0)$-space with $h \neq 0$ (not $K$-paracontact or paraSasakian). This last case is possible because (3.4) does not imply $h=0$, as can be seen in Examples 3.8-3.11.

Let us now see examples of all the possible constant ranks of $h$ that appear in Theorem 3.2. First of all, if $h=0$, the standard examples of paraSasakian manifolds are the hyperboloids

$$
\mathbb{H}_{n+1}^{2 n+1}(1)=\left\{\left(x_{0}, y_{0}, \ldots, x_{n}, y_{n}\right) \in \mathbb{R}^{2 n+2} \mid x_{0}^{2}+\ldots+x_{n}^{2}-y_{0}^{2}-\ldots-y_{n}^{2}=1\right\}
$$

and the hyperbolic Heisenberg group $\mathcal{H}^{2 n+1}=\mathbb{R}^{2 n} \times \mathbb{R}$ with the structures defined in [11]. Other examples of ( $\eta$-Einstein) paraSasakian manifolds can be obtained from contact $(\kappa, \mu)$-spaces with $\left|1-\frac{\mu}{2}\right|<\sqrt{1-\kappa}$, as seen in Theorem 3.4 of [6]. In particular, it was shown that the tangent sphere bundle $T_{1} N$ of any space form $N(c)$ with $c<0$ admits a canonical $\eta$-Einstein paraSasakian structure.

We can also construct paraSasakian examples by defining a paracontact metric structure on a Lie group:

Example 3.4. (Canonical paraSasakian structure on the Heisenberg algebra). Let $\mathfrak{g}$ be the $(2 n+1)$-dimensional Lie algebra with basis $\left\{\xi, X_{1}, \ldots, X_{2 n}\right\}$ such that the only non-vanishing Lie brackets are

$$
\left[X_{2 i-1}, X_{2 i}\right]=2 \xi, \quad i=1, \ldots, n \text {. }
$$


If we denote by $G$ the Lie group whose Lie algebra is $\mathfrak{g}$, we can define a left-invariant paracontact metric structure on $G$ the following way:

$$
\begin{gathered}
\varphi \xi=0, \quad \varphi X_{2 i-1}=X_{2 i}, \quad \varphi X_{2 i}=X_{2 i-1}, \quad i=1, \ldots, n, \\
\eta(\xi)=1, \quad \eta\left(X_{i}\right)=0, \quad i=1, \ldots, 2 n,
\end{gathered}
$$

the only non-vanishing components of the metric are

$$
g(\xi, \xi)=g\left(X_{2 i}, X_{2 i}\right)=1, \quad g\left(X_{2 i-1}, X_{2 i-1}\right)=-1, \quad i=1, \ldots, n .
$$

A straightforward computation gives that $h=0$. Moreover, using properties of paracontact metric manifolds and Koszul's formula, we obtain that

$$
\begin{gathered}
\nabla_{X_{2 i}} \xi=-X_{2 i-1}, \quad \nabla_{X_{2 i-1}} \xi=-X_{2 i}, \quad i=1, \ldots, n \\
\nabla_{X_{2 i}} X_{2 i}=\nabla_{X_{2 i-1}} X_{2 i-1}=0, \quad \nabla_{X_{2 i-1}} X_{2 j}=-\nabla_{X_{2 i}} X_{2 j-1}=\delta_{i j} \xi, \quad i=1, \ldots, n .
\end{gathered}
$$

Therefore,

$$
\begin{aligned}
R\left(X_{i}, \xi\right) \xi & =-X_{i}, \quad i=1, \ldots, 2 n, \\
R\left(X_{i}, X_{j}\right) \xi & =0, \quad i, j=1, \ldots, 2 n .
\end{aligned}
$$

We conclude that $R(X, Y) \xi=-(\eta(Y) X-\eta(X) Y)$ for all $X, Y$ on $M$, thus the manifold is paraSasakian because of Theorem 3.1. Alternatively, we could check the normality condition by proving that $[\varphi, \varphi](X, Y)-2 d \eta(X, Y) \xi=0$ for all vector fields $X, Y$ on $M$.

If we apply a $D_{c}$-homothetic deformation to any of the previous paraSasakian examples, we obtain again paraSasakian manifolds ([15]).

Let us now construct some examples of paracontact metric $(-1, \mu)$-spaces with $h \neq 0$. We will begin by adapting some paracontact metric $(-1,2)$-spaces with $h \neq 0$ that appear in the literature and will afterwards construct explicitly $(2 n+1)$-dimensional paracontact metric $(-1,2)$-spaces with $h \neq 0$ such that rank of $h$ attains all values in $\{1, \ldots, n\}$. Lastly, the case $\mu \neq 2$ will be discussed.

Example 3.5. $((2 n+1)$-dimensional paracontact metric $(-1,2)$-space, $\operatorname{rank}(h)=$ $n)$. We will begin with the examples that were presented in [8]. Let us take a flat Riemannian manifold $M$ and construct on it the tangent sphere bundle $T_{1} M$ with its standard contact metric structure $(\varphi, \xi, \eta, g)$, which satisfies $R(X, Y) \xi=0$ for every $X, Y$ on $T_{1} M$ (see [2]). Then we can define a new structure by taking

$$
\varphi_{2}=h, \quad g_{2}=d \eta(\cdot, h \cdot)+\eta \otimes \eta,
$$


which is a paracontact metric $(-1,2)$-space (Theorem 3.4 of [8]). We will now see the form that $h_{2}$ has on these examples.

Let us first take a $\varphi$-basis $\left\{\xi, X_{1}, \varphi X_{1}, \ldots, X_{n}, \varphi X_{n}\right\}$ of the contact metric $(\kappa, \mu)$ space such that $h X_{i}=X_{i}$ and $h \varphi X_{i}=-\varphi X_{i}$ (which exists because of [2]). Then $\left\{\xi, \widetilde{X}_{1}, \widetilde{Y}_{1}, \ldots, \widetilde{X}_{n}, \widetilde{Y}_{n}\right\}$, where $\widetilde{X}_{i}:=\frac{1}{\sqrt{2}} X_{i}, \widetilde{Y}_{i}:=\sqrt{2} \varphi X_{i}$, is a basis for which the only non-vanishing components of the metric $g_{2}$ are

$$
g_{2}(\xi, \xi)=1, \quad g_{2}\left(\widetilde{X}_{i}, \widetilde{Y}_{i}\right)=1,
$$

the tensor $\varphi_{2}$ satisfies

$$
\varphi_{2} \widetilde{X}_{i}=\widetilde{X}_{i}, \quad \varphi_{2} \widetilde{Y}_{i}=-\widetilde{Y}_{i}
$$

and

$$
h_{2 \mid\left\langle\widetilde{X}_{i}, \widetilde{Y}_{i}\right\rangle}=\left(\begin{array}{cc}
0 & 0 \\
1 & 0
\end{array}\right)
$$

for all $i=1, \ldots, n$.

Therefore,

$$
h_{2}=\left(\begin{array}{ccccccc}
0 & & & & & \\
& 0 & 0 & & & \\
& 1 & 0 & & & \\
& & & \ddots & & \\
& & & & 0 & 0 \\
& & & & 1 & 0
\end{array}\right)
$$

so $\operatorname{rank}\left(h_{2}\right)=n$.

In dimension 3, we also have Example 6.2 from [13], which is a 3-dimensional paracontact metric $(-1,2)$-space with $h \neq 0$ satisfying (3.1).

On the other hand, we can give examples using left-invariant paracontact metric structures on Lie groups of $(2 n+1)$-dimensional paracontact metric $(-1,2)$-spaces with $h \neq 0$ and $\operatorname{rank}(h)=m \in\{1, \ldots, n\}$.

Example 3.6. $((2 n+1)$-dimensional paracontact metric $(-1,2)$-space with $\operatorname{rank}(h)$ $=m \in\{1, \ldots, n\})$. Let $\mathfrak{g}$ be the $(2 n+1)$-dimensional Lie algebra with basis $\left\{\xi, X_{1}, Y_{1}, \ldots, X_{n}, Y_{n}\right\}$ such that the only non-vanishing components are

$$
\begin{aligned}
& {\left[\xi, X_{i}\right]=Y_{i}, \quad\left[X_{i}, Y_{j}\right]} \\
& =\left\{\begin{array}{l}
\delta_{i j}\left(2 \xi+\sqrt{2}\left(1+\delta_{i m}\right) Y_{m}\right)+\left(1-\delta_{i j}\right) \sqrt{2}\left(\delta_{i m} Y_{j}+\delta_{j m} Y_{i}\right), \quad i, j=1, \ldots, m, \\
\delta_{i j}\left(2 \xi+\sqrt{2} Y_{i}\right), \quad i, j=m+1, \ldots, n, \\
\sqrt{2} Y_{i} \quad i=1, \ldots, m, j=m+1, \ldots, n .
\end{array}\right.
\end{aligned}
$$


If we denote by $G$ the Lie group whose Lie algebra is $\mathfrak{g}$, we can define a leftinvariant paracontact metric structure on $G$ the following way:

$$
\begin{gathered}
\varphi \xi=0, \quad \varphi X_{i}=X_{i}, \quad \varphi Y_{i}=-Y_{i}, \quad i=1, \ldots, n, \\
\eta(\xi)=1, \quad \eta\left(X_{i}\right)=\eta\left(Y_{i}\right)=0, \quad i=1, \ldots, n .
\end{gathered}
$$

The only non-vanishing components of the metric are

$$
g(\xi, \xi)=g\left(X_{i}, Y_{i}\right)=1, \quad i=1, \ldots, n .
$$

A straightforward computation gives that $h X_{i}=Y_{i}$ if $i=1, \ldots, m, h X_{i}=0$ if $i=m+1, \ldots, n$ and $h Y_{j}=0$ if $j=1, \ldots, n$, so $h^{2}=0$ and $\operatorname{rank}(h)=m$.

Moreover, by basic paracontact metric properties we obtain that $\nabla_{X_{i}} \xi=-X_{i}-Y_{i}$ if $i=1, \ldots, m, \nabla_{X_{i}} \xi=-X_{i}$ if $i=m+1, \ldots, n$ and $\nabla_{Y_{i}} \xi=Y_{i}$ if $i=1, \ldots, n$, from which we deduce that

$$
\begin{aligned}
R\left(X_{i}, \xi\right) \xi & =-X_{i}+2 Y_{i}=-X_{i}+2 h X_{i}, \quad i=1, \ldots, m, \\
R\left(X_{i}, \xi\right) \xi & =-X_{i}, \quad i=m+1, \ldots, n, \\
R\left(Y_{i}, \xi\right) \xi & =-Y_{i}, \quad i=1, \ldots, n .
\end{aligned}
$$

Therefore, $R(X, \xi) \xi=-X+2 h X$ for every $X$ orthogonal to $\xi$.

Using Koszul's formula, we can compute $\nabla_{X_{i}} Y_{j}$ and $\nabla_{Y_{i}} Y_{j}$ for any $i, j$ :

$$
\begin{aligned}
& \nabla_{X_{i}} Y_{j}= \begin{cases}\delta_{i j}\left(\xi+\sqrt{2}\left(1+\delta_{i m} Y_{m}\right)\right) & \\
\quad+\left(1-\delta_{i j}\right) \sqrt{2}\left(\delta_{i m} Y_{j}+\delta_{j m} Y_{i}\right), & i, j=1, \ldots, m, \\
\sqrt{2} Y_{i}, & i=1, \ldots, m, j=m+1, \ldots, n, \\
0, & i=m+1, \ldots, n, j=1, \ldots, m, \\
\delta_{i j}\left(\xi+\sqrt{2} Y_{i}\right), & i, j=m+1, \ldots, n,\end{cases} \\
& \nabla_{Y_{i} Y_{j}}=0, \quad i, j=1, \ldots, n .
\end{aligned}
$$

It follows that $R\left(X_{i}, X_{j}\right) \xi=R\left(X_{i}, Y_{j}\right) \xi=R\left(Y_{i}, Y_{j}\right) \xi=0$ for any $i, j=1, \ldots, n$, which is enough to conclude that the manifold is a $(-1,2)$-space.

If we take $m=0$ in the previous example, we will obtain a $(2 n+1)$-dimensional paracontact metric $(-1,2)$-space such that $\operatorname{rank}(h)=n$, as in Example 3.5. However, the construction of our manifold has been considerably different.

We can also change the Lie algebra and paracontact structure of Example 3.6 to obtain other possibilities. For example, in the particular case $m=1$, we have the following one. 
Example 3.7. $((2 n+1)$-dimensional paracontact metric $(-1,2)$-space with rank $(h)=1)$. Let $\mathfrak{g}$ be the $(2 n+1)$-dimensional Lie algebra with basis $\left\{\xi, X_{1}, Y_{1}, \ldots, X_{n}\right.$, $\left.Y_{n}\right\}$ such that the only non-vanishing components are

$$
\begin{gathered}
{\left[\xi, X_{1}\right]=Y_{1}, \quad\left[X_{1}, Y_{1}\right]=2 \xi} \\
{\left[X_{i}, Y_{i}\right]=2\left(\xi+X_{i}\right), \quad\left[X_{1}, X_{i}\right]=Y_{1}, \quad i=2, \ldots, n .}
\end{gathered}
$$

If we denote by $G$ the Lie group whose Lie algebra is $\mathfrak{g}$, we can define a leftinvariant paracontact metric structure on $G$ the following way:

$$
\begin{gathered}
\varphi \xi=0, \quad \varphi X_{1}=X_{1}, \quad \varphi Y_{1}=-Y_{1}, \quad \varphi X_{i}=-X_{i}, \quad \varphi Y_{i}=Y_{i}, \quad i=2, \ldots, n, \\
\eta(\xi)=1, \quad \eta\left(X_{i}\right)=\eta\left(Y_{i}\right)=0, \quad i=1, \ldots, n .
\end{gathered}
$$

The only non-vanishing components of the metric are

$$
g(\xi, \xi)=g\left(X_{1}, Y_{1}\right)=1, \quad g\left(X_{i}, Y_{i}\right)=-1, \quad i=2, \ldots, n .
$$

A straightforward computation gives that $h X_{1}=Y_{1}, h Y_{1}=0$ and $h X_{i}=h Y_{i}=0$, $i=2, \ldots, n$, so $h^{2}=0$ and $\operatorname{rank}(h)=1$.

Moreover, by basic paracontact metric properties and Koszul's formula we obtain that

$$
\begin{aligned}
& \nabla_{\xi} X_{1}=-X_{1}, \quad \nabla_{\xi} Y_{1}=Y_{1}, \quad \nabla_{\xi} X_{i}=X_{i}, \quad \nabla_{\xi} Y_{i}=-Y_{i}, \quad i=2, \ldots, n, \\
& \nabla_{Y_{i}} Y_{j}=2 \delta_{i j}\left(1-\delta_{i 1}\right) Y_{i}, \quad \nabla_{X_{i}} Y_{j}=\delta_{i j} \xi \\
& \nabla_{Y_{i}} X_{j}=\delta_{i j}\left(-\xi+2\left(1-\delta_{i 1}\right) X_{i}\right), \quad i, j=1, \ldots, n \\
& \nabla_{X_{i}} X_{1}=0, \quad \nabla_{X_{1}} X_{j}=Y_{1}, \quad i=2, \ldots, n,
\end{aligned}
$$

which is enough to prove that

$$
\begin{aligned}
R\left(X_{1}, \xi\right) \xi & =-X_{1}+2 Y_{1}=-X_{1}+2 h X_{1}, \\
R\left(X_{i}, \xi\right) \xi & =-X_{i}, \quad i=2, \ldots, n \\
R\left(Y_{i}, \xi\right) \xi & =-Y_{i}, \quad i=1, \ldots, n \\
R\left(X_{i}, X_{j}\right) \xi & =R\left(X_{i}, Y_{j}\right) \xi=R\left(Y_{i}, Y_{j}\right) \xi=0, \quad i, j=1, \ldots, n .
\end{aligned}
$$

Therefore, the manifold is also a $(-1,2)$-space.

Note that all the examples constructed until now and most of the ones appearing in the literature of paracontact metric $(-1, \mu)$-spaces with $h^{2}=0$ but $h \neq 0$ have $\mu=2$. Why is this particular value so important? First of all, given a non-Sasakian contact 
metric $(\kappa, \mu)$-space, we can define two canonical paracontact metric structures on $M$, $\left(\widetilde{\varphi}_{1}, \xi, \eta, \widetilde{g}_{1}\right)$ and $\left(\widetilde{\varphi}_{2}, \xi, \eta, \widetilde{g}_{2}\right)$, in the following way ([5] and [7]):

$$
\begin{array}{lll}
\widetilde{\varphi}_{1}:=\frac{1}{\sqrt{1-\kappa}} \varphi h, & \widetilde{g}_{1}:=\frac{1}{\sqrt{1-\kappa}} g(\cdot, h \cdot)+\eta \otimes \eta, \\
\widetilde{\varphi}_{2}:=\frac{1}{\sqrt{1-\kappa}} h, & \widetilde{g}_{2}:=\frac{1}{\sqrt{1-\kappa}} g(\cdot, \varphi h \cdot)+\eta \otimes \eta .
\end{array}
$$

Moreover, these new structures are paracontact metric $\left(\widetilde{\kappa}_{i}, \widetilde{\mu}_{i}\right)$-spaces with

$$
\begin{array}{lll}
\widetilde{\kappa}_{1}=\left(1-\frac{\mu}{2}\right)^{2}-1, & \widetilde{\mu}_{1}=2(1-\sqrt{1-\kappa}), & \widetilde{h}_{1}=-\frac{1-\frac{\mu}{2}}{\sqrt{1-\kappa}} h, \\
\widetilde{\kappa}_{2}=\kappa-2+\left(1-\frac{\mu}{2}\right)^{2}, & \widetilde{\mu}_{2}=2, & \widetilde{h}_{2}=\frac{1-\frac{\mu}{2}}{\sqrt{1-\kappa}} \varphi h+\sqrt{1-\kappa} \varphi .
\end{array}
$$

Therefore, $\widetilde{\kappa}_{1}=-1$ if and only if $\mu=2$, which is equivalent to $\left(\widetilde{\varphi}_{1}, \xi, \eta, \widetilde{g}_{1}\right)$ being paraSasakian ([5, Th. 5.9] or Theorem 3.2). On the other hand, $\widetilde{\kappa}_{2}=-1$ if and only if $\sqrt{1-\kappa}=1-\frac{\mu}{2}$, from which it follows that $h_{2}=\varphi h+\left(1-\frac{\mu}{2}\right) \varphi$. This means that the only possibility of constructing a paracontact metric $(\widetilde{\kappa}, \widetilde{\mu})$-space with $\widetilde{h}^{2}=0$ and $\widetilde{h} \neq 0$ using these structures is to take the second one, which has $\widetilde{\mu}=2$.

Another reason for which the value $\mu=2$ is special comes from the fact that applying a $D_{c}$-homothetic deformation to a paracontact metric $(-1,2)$-space gives us another paracontact metric $(-1,2)$-space for any real $c \neq 0$.

What happens when $\mu \neq 2$ ? Are there any examples of paracontact metric $(-1, \mu)$ spaces with $h \neq 0$ and $\mu \neq 2$ ? The answer is yes, as was shown in Example 4.6 of [10] for the 3-dimensional case. We will provide proof in dimensions greater than 3 in Examples 3.8-3.11. Before constructing them, note that, given a $(-1, \mu)$-space with $\mu \neq 2$, a $\mathcal{D}_{c}$-homothetic deformation with $c=1-\frac{\mu}{2} \neq 0$ will give us a paracontact metric $(-1,0)$-space thanks to (2.4). Conversely, given a paracontact metric $(-1,0)$ space, if we $\mathcal{D}_{c}$-homothetically deform it with $c=\frac{2}{2-\mu} \neq 0$ for some $\mu \neq 2$, we will obtain a paracontact metric $(-1, \mu)$-space with $\mu \neq 2$.

Lastly, the rank of $h$ and $h^{\prime}$ coincides because $h^{\prime}=\frac{1}{c} h$ ([8]) and a simple change of basis will give us another one satisfying all the properties of Theorem 3.2. Therefore, it makes sense to concentrate on the $\mu=0$ case, which is also special because the paracontact metric $(-1,0)$-spaces with $h \neq 0$ satisfy (2.1) but are not paraSasakian manifolds.

We can give examples using left-invariant paracontact metric structures on Lie groups of $(2 n+1)$-dimensional paracontact metric $(-1,0)$-spaces with $h \neq 0$. We recall that $\operatorname{rank}\left(h_{p}\right) \in\{1, \ldots, n\}$ (Theorem 3.2) and we will first see the case $\operatorname{rank}(h)=1$.

Example 3.8. $((2 n+1)$-dimensional paracontact metric $(-1,0)$-space with $\operatorname{rank}(h)$ $=1$ ). Let $\mathfrak{g}$ be the $(2 n+1)$-dimensional Lie algebra with basis $\left\{\xi, X_{1}, Y_{1}, \ldots, X_{n}, Y_{n}\right\}$ 
such that the only non-vanishing components are

$$
\begin{gathered}
{\left[\xi, X_{1}\right]=X_{1}+Y_{1}, \quad\left[\xi, Y_{1}\right]=-Y_{1}, \quad\left[X_{1}, Y_{1}\right]=2 \xi} \\
{\left[X_{i}, Y_{i}\right]=2\left(\xi+Y_{i}\right), \quad\left[X_{1}, Y_{i}\right]=X_{1}+Y_{1}, \quad\left[Y_{1}, Y_{i}\right]=-Y_{1}, \quad i=2, \ldots, n .}
\end{gathered}
$$

If we denote by $G$ the Lie group whose Lie algebra is $\mathfrak{g}$, we can define a leftinvariant paracontact metric structure on $G$ the following way:

$$
\begin{gathered}
\varphi \xi=0, \quad \varphi X_{1}=X_{1}, \quad \varphi Y_{1}=-Y_{1}, \quad \varphi X_{i}=-X_{i}, \quad \varphi Y_{i}=Y_{i}, \quad i=2, \ldots, n \\
\eta(\xi)=1, \quad \eta\left(X_{i}\right)=\eta\left(Y_{i}\right)=0, \quad i=1, \ldots, n
\end{gathered}
$$

The only non-vanishing components of the metric are

$$
g(\xi, \xi)=g\left(X_{1}, Y_{1}\right)=1, \quad g\left(X_{i}, Y_{i}\right)=-1, \quad i=2, \ldots, n .
$$

A straightforward computation gives that $h X_{1}=Y_{1}, h Y_{1}=0$ and $h X_{i}=h Y_{i}=0$, $i=2, \ldots, n$, so $h^{2}=0$ and $\operatorname{rank}(h)=1$.

Moreover, by basic paracontact metric properties and Koszul's formula we obtain that

$$
\begin{aligned}
& \nabla_{\xi} X_{1}=0, \quad \nabla_{\xi} Y_{1}=0, \quad \nabla_{\xi} X_{i}=X_{i}, \quad \nabla_{\xi} Y_{i}=-Y_{i}, \quad i=2, \ldots, n, \\
& \nabla_{X_{i}} Y_{1}=\delta_{i 1} \xi, \quad \nabla_{X_{i}} Y_{j}=\delta_{i j}\left(\xi+2 Y_{i}\right) \\
& \nabla_{Y_{1}} X_{1}=-\xi, \nabla_{Y_{i}} X_{j}=-\delta_{i j} \xi, \quad i, j=2, \ldots, n, \\
& \nabla_{X_{1}} X_{j}=0, \nabla_{Y_{1}} Y_{1}=\nabla_{Y_{1}} Y_{j}=0, \quad \nabla_{Y_{j}} Y_{1}=Y_{1}, \quad i=2, \ldots, n,
\end{aligned}
$$

and thus

$$
\begin{aligned}
R\left(X_{i}, \xi\right) \xi & =-X_{i}, \quad i=1, \ldots, n, \\
R\left(Y_{i}, \xi\right) \xi & =-Y_{i}, \quad i=1, \ldots, n, \\
R\left(X_{i}, X_{j}\right) \xi & =R\left(X_{i}, Y_{j}\right) \xi=R\left(Y_{i}, Y_{j}\right) \xi=0, \quad i, j=1, \ldots, n .
\end{aligned}
$$

Therefore, the manifold is also a $(-1,0)$-space.

To our knowledge, the previous example is the first paracontact metric $(-1, \mu)$ space with $h^{2}=0, h \neq 0$ and $\mu \neq 2$ that has been constructed in dimensions greater than 3. For dimension 3, Example 4.6 of [10] was already known.

Let us now see some other possibilities. In dimension $3, n=1$, so the previous example gives the only possible value of the rank of $h$. In dimension 5 , we can construct an example with $\operatorname{rank}(h)=2$ in the following way: 
Example 3.9. (5-dimensional paracontact metric $(-1,0)$-space with $\operatorname{rank}(h)=2)$. Let us take the 5 -dimensional Lie algebra $\mathfrak{g}$ of basis $\left\{\xi, X_{1}, Y_{1}, X_{2}, Y_{2}\right\}$ and whose Lie brackets are

$$
\begin{aligned}
{\left[\xi, X_{1}\right] } & =Y_{1}+X_{1}+X_{2}, & {\left[\xi, Y_{1}\right] } & =-Y_{1}+Y_{2}, \\
{\left[\xi, X_{2}\right] } & =Y_{2}+X_{1}+X_{2}, & {\left[\xi, Y_{2}\right] } & =Y_{1}-Y_{2}, \\
{\left[X_{1}, Y_{1}\right] } & =2 \xi+X_{2}+\frac{3}{2} Y_{2}, & {\left[X_{2}, Y_{2}\right] } & =-2 \xi+X_{1}-\frac{1}{2} Y_{1}, \\
{\left[X_{1}, X_{2}\right] } & =\frac{3}{2} X_{1}+\frac{1}{2} X_{2}, & {\left[Y_{1}, Y_{2}\right] } & =-Y_{1}+Y_{2}, \\
{\left[X_{1}, Y_{2}\right] } & =\frac{3}{2} Y_{1}+X_{2}, & {\left[Y_{1}, X_{2}\right] } & =-X_{1}+\frac{1}{2} Y_{2} .
\end{aligned}
$$

If we denote by $G$ the Lie group whose Lie algebra is $\mathfrak{g}$, we can define on it a leftinvariant paracontact metric structure with

$$
\varphi \xi=0, \quad \varphi X_{i}=X_{i}, \quad \varphi Y_{i}=-Y_{i}, \quad \eta(\xi)=1, \quad \eta\left(X_{i}\right)=\eta\left(Y_{i}\right)=0, \quad i=1,2 .
$$

and whose only non-vanishing components of the metric are

$$
g(\xi, \xi)=g\left(X_{1}, Y_{1}\right)=1, \quad g\left(X_{2}, Y_{2}\right)=-1 .
$$

We can check that $h X_{i}=Y_{i}$ and $h Y_{i}=0, i=1,2$, so $h^{2}=0$ and $\operatorname{rank}(h)=2$.

Moreover, long but straightforward computations give us that the manifold is a $(-1,0)$-space.

We show below examples of dimension 7 with $\operatorname{rank}(h)=2$ and $\operatorname{rank}(h)=3$, respectively. Higher-dimensional examples could be constructed analogously.

Example 3.10. (7-dimensional paracontact metric $(-1,0)$-space with $\operatorname{rank}(h)=2$ ). Let us define a 7 -dimensional Lie algebra $\mathfrak{g}$ of basis $\left\{\xi, X_{1}, Y_{1}, X_{2}, Y_{2}, X_{3}, Y_{3}\right\}$ whose only non-vanishing Lie brackets are

$$
\begin{aligned}
{\left[\xi, X_{1}\right] } & =\left[X_{1}, X_{3}\right]=Y_{1}+X_{1}+X_{2}, & {\left[\xi, Y_{1}\right] } & =\left[Y_{1}, X_{3}\right]=-Y_{1}+Y_{2}, \\
{\left[\xi, X_{2}\right] } & =\left[X_{2}, X_{3}\right]=Y_{2}+X_{1}+X_{2}, & {\left[\xi, Y_{2}\right] } & =\left[Y_{2}, X_{3}\right]=Y_{1}-Y_{2}, \\
{\left[X_{1}, Y_{1}\right] } & =2 \xi+\sqrt{2}\left(X_{2}+Y_{2}\right), & {\left[X_{2}, Y_{2}\right] } & =-2 \xi+\sqrt{2} X_{1}, \\
{\left[X_{3}, Y_{3}\right] } & =-2 \xi-2 X_{3}, & {\left[X_{1}, X_{2}\right] } & =-\left[Y_{1}, X_{2}\right]=\sqrt{2} X_{1}, \\
{\left[X_{1}, Y_{2}\right] } & =\sqrt{2}\left(Y_{1}+X_{2}\right), & {\left[Y_{1}, Y_{2}\right] } & =\sqrt{2}\left(-Y_{1}+Y_{2}\right) .
\end{aligned}
$$

If we denote by $G$ the Lie group whose Lie algebra is $\mathfrak{g}$, we can define on it a leftinvariant paracontact metric structure with $\varphi \xi=0, \quad \varphi X_{i}=X_{i}, \quad \varphi Y_{i}=-Y_{i}, \quad \eta(\xi)=1, \quad \eta\left(X_{i}\right)=\eta\left(Y_{i}\right)=0, \quad i=1,2,3$. 
and whose only non-vanishing components of the metric are

$$
g(\xi, \xi)=g\left(X_{1}, Y_{1}\right)=1, \quad g\left(X_{2}, Y_{2}\right)=g\left(X_{3}, Y_{3}\right)=-1 .
$$

A direct computation gives that $h X_{i}=Y_{i}, i=1,2, h X_{3}=0$ and $h Y_{i}=0, i=1,2,3$, so $h^{2}=0$ and $\operatorname{rank}(h)=2$.

Moreover, long but straightforward computations give us that the manifold is a $(-1,0)$-space.

Example 3.11. (7-dimensional paracontact metric $(-1,0)$-space with $\operatorname{rank}(h)=3)$. Let us define a 7-dimensional Lie algebra $\mathfrak{g}$ of basis $\left\{\xi, X_{1}, Y_{1}, X_{2}, Y_{2}, X_{3}, Y_{3}\right\}$ whose Lie brackets are

$$
\begin{aligned}
{\left[\xi, X_{1}\right] } & =Y_{1}+X_{1}+X_{2}, & {\left[\xi, Y_{1}\right] } & =-Y_{1}+Y_{2}, \\
{\left[\xi, X_{2}\right] } & =Y_{2}+X_{1}+X_{2}, & {\left[\xi, Y_{2}\right] } & =Y_{1}-Y_{2}, \\
{\left[\xi, X_{3}\right] } & =X_{3}+Y_{3}, & {\left[\xi, Y_{3}\right] } & =-Y_{3}, \\
{\left[X_{1}, Y_{1}\right] } & =2 \xi+\sqrt{2}\left(X_{2}+Y_{2}\right), & {\left[X_{2}, Y_{2}\right] } & =-2 \xi+\sqrt{2} X_{1}, \\
{\left[X_{3}, Y_{3}\right] } & =-2 \xi+\sqrt{2}\left(X_{1}-X_{2}-Y_{2}\right), & {\left[X_{1}, X_{2}\right] } & =-\left[Y_{1}, X_{2}\right]=\sqrt{2} X_{1}, \\
{\left[X_{1}, Y_{2}\right] } & =\sqrt{2}\left(Y_{1}+X_{2}\right), & {\left[Y_{1}, Y_{2}\right] } & =\sqrt{2}\left(-Y_{1}+Y_{2}\right), \\
{\left[X_{1}, Y_{3}\right] } & =-\left[X_{2}, X_{3}\right]=\left[X_{2}, Y_{3}\right]=\sqrt{2} X_{3}, & {\left[Y_{1}, Y_{3}\right] } & =-\left[Y_{2}, X_{3}\right]=\left[Y_{2}, Y_{3}\right]=\sqrt{2} Y_{3} .
\end{aligned}
$$

If we denote by $G$ the Lie group whose Lie algebra is $\mathfrak{g}$, we can define on it a leftinvariant paracontact metric structure with

$$
\varphi \xi=0, \quad \varphi X_{i}=X_{i}, \quad \varphi Y_{i}=-Y_{i}, \quad \eta(\xi)=1, \quad \eta\left(X_{i}\right)=\eta\left(Y_{i}\right)=0, \quad i=1,2,3 .
$$

and whose only non-vanishing components of the metric are

$$
g(\xi, \xi)=g\left(X_{1}, Y_{1}\right)=1, \quad g\left(X_{2}, Y_{2}\right)=g\left(X_{3}, Y_{3}\right)=-1 .
$$

Therefore, $h X_{i}=Y_{i}, i=1,2,3$ and $h Y_{i}=0, i=1,2,3$, thus $h^{2}=0$ and $\operatorname{rank}(h)=3$. Moreover, direct computations give us that the manifold is a $(-1,0)$-space.

Remark 3.12. It is worth mentioning that Theorem 3.2 is true only pointwise, i.e. $\operatorname{rank}\left(h_{p}\right)$ does not need to be the same for every $p \in M$. However, there are no known examples of paracontact metric $(-1, \mu)$-spaces where $\operatorname{rank}(h)$ is not constant.

$$
\text { 4. AnotherManifold with } h^{2}=0 \text { But } h \neq 0
$$

Lastly, we would like to remark that, although the first examples of $h^{2}=0$ but $h \neq 0$ appeared (quite naturally) in the context of paracontact metric $(\kappa, \mu)$-spaces, it is not difficult to construct paracontact metric manifolds satisfying these properties which are not $(-1, \mu)$-spaces. 
Example 4.1. Let us take the 5-dimensional Lie algebra $\mathfrak{g}$ of basis $\left\{\xi, X_{1}, Y_{1}, X_{2}\right.$, $Y_{2}$ \} such that the only non-vanishing Lie brackets are

$$
\left[\xi, X_{1}\right]=Y_{1}, \quad\left[X_{1}, Y_{1}\right]=2 \xi, \quad\left[X_{2}, Y_{2}\right]=2\left(\xi+X_{2}\right), \quad\left[X_{1}, X_{2}\right]=Y_{1} .
$$

If we denote by $G$ the Lie group whose Lie algebra is $\mathfrak{g}$, we can define on it a leftinvariant paracontact metric structure:

$$
\varphi \xi=0, \quad \varphi X_{i}=X_{i}, \quad \varphi Y_{i}=-Y_{i}, \quad \eta(\xi)=1, \quad \eta\left(X_{i}\right)=\eta\left(Y_{i}\right)=0, \quad i=1,2 .
$$

The only non-vanishing components of the metric are

$$
g(\xi, \xi)=g\left(X_{1}, Y_{1}\right)=g\left(X_{2}, Y_{2}\right)=1 .
$$

Therefore, $h X_{1}=Y_{1}$ and $h Y_{1}=h X_{2}=h Y_{2}=0$, so $h^{2}=0$ but $h \neq 0$.

Although $R(X, \xi) \xi=-X+2 h X$ for all vector field $X$ orthogonal to $\xi$, we can check that $R\left(X_{1}, X_{2}\right) \xi=-2 Y_{1} \neq 0$, so the manifold is not a $(-1,2)$-space.

Remark 4.2. Note that the previous Lie algebra coincides with the one of Example 3.7 for $n=2$ (hence the form of $h$ coincides) but that the construction of the paracontact metric structure is not the same, since both $\varphi$ and $g$ are defined differently.

\section{ACKNOWLEDGMENTS}

The author would like to thank Prof. Martín Avendaño for his valuable suggestions and insights and the referee for his great knowledge and expertise on the field of paracontact geometry.

\section{REFERENCES}

1. D. E. Blair, Riemannian Geometry of Contact and Symplectic Manifolds, Second Edition, Progress in Mathematics 203, Birkhäuser, Boston, 2010.

2. D. E. Blair, T. Koufogiorgos and B. J. Papantoniou, Contact metric manifolds satisfyng a nullity condition, Israel J. Math., 91 (1995), 189-214.

3. E. Boeckx, A full classification of contact metric $(\kappa, \mu)$-spaces, Illinois J. Math., 44 (2000), 212-219.

4. B. Cappelletti Montano, Bi-Legendrian structures and paracontact geometry, Int. J. Geom. Met. Mod. Phys., 6 (2009), 487-504.

5. B. Cappelletti Montano, Bi-paracontact structures and Legendre foliations, Kodai Math. J., 33 (2010), 473-512.

6. B. Cappelletti Montano, A. Carriazo and V. Martín-Molina, Sasaki-Einstein and paraSasakiEinstein metrics from $(\kappa, \mu)$-structures, J. Geom. Physics., 73 (2013) 20-36. 
7. B. Cappelletti Montano and L. Di Terlizzi, Geometric structures associated with a contact metric $(\kappa, \mu)$-space, Pacific J. Math., 246 (2010), 257-292.

8. B. Cappelletti Montano, I. Küpeli Erken and C. Murathan, Nullity conditions in paracontact geometry, Differential Geom. Appl., 30 (2012), 665-693.

9. G. Calvaruso, Homogeneous paracontact metric three-manifolds, Illinois J. Math., 55 (2011), 697-718.

10. G. Calvaruso and D. Perrone, Geometry of H-paracontact Metric Manifolds, arXiv:1307. 7662.

11. S. Ivanov, D. Vassilev and S. Zamkovoy, Conformal paracontact curvature and the local flatness theorem, Geom. Dedicata, 144 (2010), 79-100.

12. S. Kaneyuki and F. L. Williams, Almost paracontact and parahodge structures on manifolds, Nagoya Math. J., 99 (1985), 173-187.

13. C. Murathan and I. Küpeli Erken, The Harmonicity of the Reeb Vector Dield on Paracontact Metric 3-manifolds, arXiv:1305.1511v2.

14. B. O’Neill, Semi-Riemannian Geometry with Applications to Relativity, Academic Press, New York, 1983.

15. S. Zamkovoy, Canonical connections on paracontact manifolds, Ann. Glob. Anal. Geom., 36 (2009), 37-60.

16. S. Zamkovoy and V. Tzanov, Non-existence of flat paracontact metric structures in dimension greater than or equal to five, Annuaire Univ. Sofia Fac. Math. Inform., 100 (2011), 27-34.

\author{
Verónica Martín-Molina \\ Centro Universitario de la Defensa \\ Academia General Militar \\ Ctra. de Huesca s/n, 50090 Zaragoza \\ and \\ I.U.M.A, Universidad de Zaragoza \\ Spain \\ E-mail: vmartin@unizar.es
}

